\title{
IRON AND CALCIUM CONTENT OF EDIBLE PLANTS IN NON CONVENTIONAL FAMILY SOLANACEAE: A COMPARATIVE ANALYSIS
}

\author{
MARANGON, Cristiane ${ }^{1}$; RIZZATTI, Ivanise Maria ${ }^{1 *}$; SOUZA, Juliane Marques de ${ }^{1}$; SANTANA \\ Jordânia Gabriela Aires ${ }^{1}$; SCHVEITZRT, Bianca ${ }^{2}$ \\ ${ }_{1}^{1}$ Universidade Estadual de Roraima, Laboratório de Turismo Ecologia e Meio Ambiente, Rua Sete de Setembro, \\ 231, cep 69301-560, Roriama - RR, Brasil \\ (fone: +55 952121 0955; fax: +55 952121 0950) \\ ${ }^{2}$ Laboratório de Ensaio Químico - Estação Experimental de Caçador - Empresa de Pesquisa Agropecuária e \\ Extensão Rural de Santa Catarina - EPAGRI, Rua Alcides Tombini, 33, cep. 89500-000, Caçador - SC, Brasil \\ (fone: +55 483665 0741; fax: +55 483665 5010) \\ ${ }^{*}$ Autor correspondente \\ e-mail: niserizzatti@gmail.com
}

Received 15 July 2015; received in revised form 1 December 2015; accepted 10 January 2016

\begin{abstract}
RESUMO
O presente artigo compara o teor de cálcio e ferro presente em três espécies de Solanaceae, são elas: Solanum paniculatum L., Solanum sessiflorum D. e Physalis angulata L., todas consideradas plantas alimentícias não-convencionais (PANC's) por serem pouco exploradas pela população em geral. As três espécies, coletadas em comunidades ribeirinhas da região do Baixo rio Branco, Roraima, tem seus teores nutricionais comparados também com plantas que são comumente utilizadas na alimentação diária, bem como com o valor de nutrientes diários recomendados pela ANVISA (agência nacional de vigilância sanitária). Como resultado verificou-se que o teor nutricional desses minerais está dentro do que é recomendado, sendo uma alternativa para reposição nutricional dos ribeirinhos, pois muitas vezes o acesso a alimentos convencionais é difícil.
\end{abstract}

Palavras-chave: Physalis angulata L., Solanum sessiliflorum D., Solanum paniculatum L., teor nutricional, Roraima.

\begin{abstract}
This study compares the amount of calcium and iron present in three species of Solanaceae, they are: Solanum paniculatum L., Solanum sessiflorum D. and Physalis angulata L., all considered unconventional food plants (PANC's) because they are unexplored by the general population. The three species were collected in riverine communities of the Lower Branco river, Roraima, and had its nutritional content compared with plants that are commonly used in the daily diet as well as the amount of daily nutrients recommended by ANVISA (National Agency of Sanitary Surveillance). As a result it was found that the nutritional content of these minerals is within what is recommended by the agency and figure as an alternative to nutritional replacement of riparian, once they usually have difficult to access conventional foods .
\end{abstract}

Keywords: Physalis angulata L., Solanum sessiliflorum D., Solanum paniculatum L., nutritional contente, Roraima.

PERIÓDICO TCHÊ QUÍMICA • www.periodico.tchequimica.com • Vol. 13 N. 25.

• ISSN 1806-0374 (impresso) • ISSN 1806-9827 (CD-ROM) • ISSN 2179-0302 (meio eletrônico)

(C) 2015. Porto Alegre, RS. Brasil 


\section{INTRODUÇÃO}

As plantas são fonte de alimento desde o início das civilizações e são usadas nas mais diversas variedades de comidas, desde a raiz até a folha. A coleta de frutos, ramos, folhas e raízes diretamente da natureza era a prática cotidiana para a obtenção de alimentos pelos antigos representantes do gênero Homo (Raven, Evert e Eichhorn,1996; Rapport, Marzoca e Drausal, 2009). Ainda, de acordo com o autor, com o desenvolvimento de técnicas de domesticação e cultivo, as plantas cultivadas alteraram-se gradualmente, por meio da seleção de características específicas de maior interesse na época.

Pode-se perceber neste momento, o começo da seleção do que se tornaria comum na alimentação tradicional dos povos e o que se tornaria não-convencional, deixado de lado, talvez pelo não conhecimento ou difícil cultivo de certas plantas. Estas últimas, conhecidas hoje por plantas alimentícias não-convencionais (PANC's) são aquelas chamadas comumente de inços, matos, sendo que algumas delas possuem um grande potencial alimentício que é, na maioria das vezes, desconhecido e por isso negligenciado (Padulosi e Hoesche-Zeledon, 2004). As PANC's são também conhecidas como hortaliças não-convencionais.

O cultivo e o consumo de muitas espécies de plantas estão deixando de ser comuns. Entretanto, o reconhecimento do potencial nutritivo das plantas alimentícias nãoconvencionais é de suma importância para o aumento da diversidade alimentar, uma vez que estas são capazes de sustentar o fornecimento de vitaminas, sais minerais e fibras para além das plantas comumente consumidas e cultivadas em maior escala.

Algumas PANC's possuem uma quantidade abundante de nutrientes em uma só fruta, folha ou raiz e poderiam substituir alguns alimentos convencionais, diminuindo assim a falta de alimentos e ingestão de nutrientes, especialmente em comunidades mais carentes e distantes dos alimentos comuns. Em comunidades com costumes mais tradicionais e afastadas dos grandes centros, o uso de plantas não-convencionais, costuma ser mais elevado do que em populações urbanas (Brasil, 2010). Esse aumento aparente pode ser relacionado à estreita relação que essas comunidades guardam com o meio e com o uso dos recursos naturais. Além disso, o frequente isolamento que essas comunidades vivem ou o baixo recurso financeiro disponível conduz essas comunidades ao uso dos recursos localmente disponíveis, seja pelo cultivo próprio, seja pela extração na floresta.

O presente estudo, desenvolvido nas comunidades ribeirinhas de Caicubi e Sacaí, na região do Baixo rio Branco, Estado de Roraima, buscou identificar e avaliar o potencial nutritivo das plantas alimentícias não-convencionais utilizadas por estas comunidades. Aqui, especificamente, apresentam-se os resultados obtidos das análises de três espécies de plantas não-convencionais comumente consumidas pelas famílias ribeirinhas. As três espécies Physalis angulata L., Solanum sessiliflorum D. e Solanum paniculatum L. (Solanaceae), tiveram o teor de Ferro ( $\mathrm{Fe})$ e Cálcio ( $\mathrm{Ca}$ ) avaliados e então comparados a outras espécies convencionais da mesma família botânica como o tomate (Solanum lycopersicum L.), o jiló (Solanum gilo Raddi) e a berinjela (Solanum melongena L.), o pimentão amarelo (Capsicum annuum L.) e a batata inglesa (Solanum tuberosum L.) comumente consumidas pela população urbana.

\section{METODOLOGIA}

As PANC's foram coletadas nas comunidades de Caicubí e Sacaí, região do baixo rio Branco, município de Caracaraí, Roraima (Figura 1). O material coletado foi herborizado, identificado e depositado no herbário do Museu Integrado de Roraima-MIRR. As análises nutricionais foram feitas no Laboratório de Ensaio Químico da EPAGRI/SC.

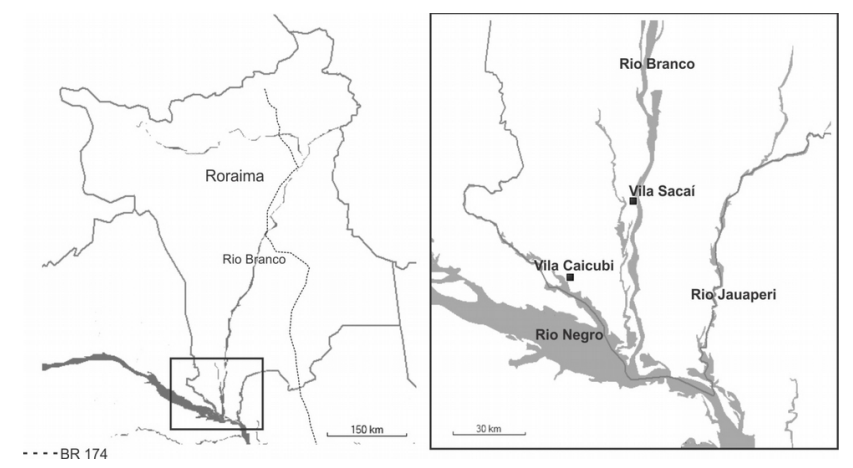

Figura 1: Mapa de localização da região do Baixo Rio Branco, estado de Roraima, Brasil. Em

PERIÓDICO TCHÊ QUÍMICA • www.periodico.tchequimica.com • Vol. 13 N. 25. • ISSN 1806-0374 (impresso) • ISSN 1806-9827 (CD-ROM) • ISSN 2179-0302 (meio eletrônico) 
detalhe, o extremo Sul de Roraima e a localização das comunidades ribeirinhas de Caicubi e Sacaí.

As pesquisas iniciais para coleta foram baseadas em técnicas etnobotânicas (Albuquerque, 2002) as quais abordam a relação homem x planta e ressaltam como diferentes grupos humanos interagem com a natureza (Amorozo e Viertler, 2002). Para a identificação das plantas como alimentícias não-convencionais foi desenvolvida uma metodologia participativa baseada no método de Diagnóstico Rural Participativo - DRP (Verdejo, 2006). A técnica utilizada foi a "caminhada com informantes" que contava com a colaboração de moradores da comunidade que se prontificaram a direcionar a equipe aos locais onde haviam plantas alimentícias não-convencionais, bem como trocar informações sobre as plantas que eles utilizam ou utilizavam na alimentação.

No percurso foi estabelecido um diálogo entre o pesquisador e os informantes voluntários, realizado de maneira informal, não dirigindo diretamente perguntas padronizadas em um roteiro, deixando-os à vontade para responder as questões levantadas. Nesta perspectiva, foi importante também não interromper as falas dos informantes para que eles se sentissem úteis e valorizados na pesquisa (Verdejo, 2006).

Depois de identificadas como PANC's as plantas foram coletadas, etiquetadas, prensadas, e secas ao sol, pois, devido à necessidade de adaptação na comunidade e ao difícil acesso à localidade, não existia uma estufa adequada para que as amostras fossem secas no local da pesquisa. No momento da coleta a preferência foi dada para amostras de ramos completos, procurando exemplares com flores e frutos em quantidade suficiente para montar uma exsicata contendo em torno de três a cinco duplicatas (Ferreira e Andrade, 2006).

No que se refere às análises químicas nos frutos foram avaliados os teores de macro e micronutrientes empregando o método utilizado para análise de polpa seca adotado pelo Laboratório de Ensaio Químico e Análise Nutricional da Epagri - SC.

Foram selecionadas amostras sadias, isentos do ataque de pragas, infecções e danos mecânicos. Assim que chegaram ao laboratório, as amostras foram secas (Estufa Marconi modelo
MA 037) com circulação forçada de ar quente a $60{ }^{\circ} \mathrm{C}$, por aproximadamente três dias. Em seguida, as amostras secas foram moídas em moinho de facas, de aço inoxidável e com peneiras de $0,5 \mathrm{~mm}$ de diâmetro, visando assegurar a completa homogeneização da amostra. Os teores minerais foram determinados por espectrometria de absorção atômica num equipamento PerkinElmer modelo AA-200, após digestão nitroperclórica, $\mathrm{HNO}_{3} / \mathrm{HClO}_{4}$ (6:1). Para construção da curva foram utilizadas soluções padronizadas Tritisol (Merck) e nas análises de cálcio empregou-se $0,1 \% \mathrm{La}$, na forma de $\mathrm{La}_{2} \mathrm{O}_{3}$.

\section{RESULTADOS E DISCUSSÃO:}

O Camapu (Physalis angulata L.) (Figura 2), utilizado pelos ribeirinhos na alimentação e na pesca, caracteriza-se por ser uma planta herbácea e ciclo anual, alcançando até um metro de altura. A produção de frutos inicia a partir do terceiro ao quarto mês e se estende por um período de até 6 meses (Lorenzo e Matos, 2002).

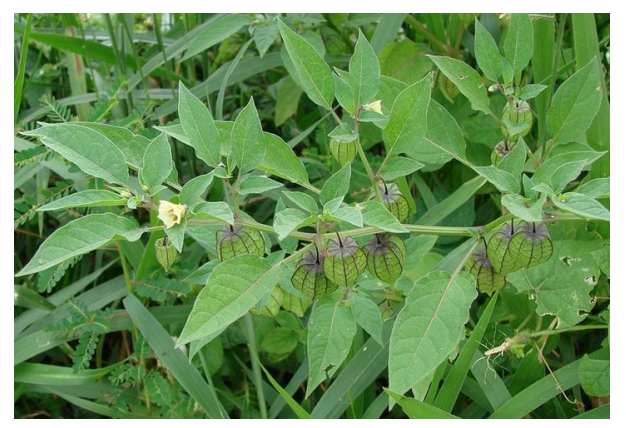

Figura 2: Camapu (Physalis angulata L.). (Aires, 2012).

Já o Cubiu (Figura 3), também conhecida como maná, topiro ou tomate de índio (Solanum sessiliflorum D.) é uma planta arbustiva nativa da Amazônia, domesticada pelas populações indígenas e que possui frutos de tamanho variável, sub-globosos a ovóides, do tipo baga, vermelho-alaranjados quando maduros, de polpa ácida com numerosas sementes achatadas (Stefanello, 2010). Seu fruto é utilizado frequente na alimentação como aperitivo, bem como na produção de geléias, doces, sucos, sorvetes e cosméticos (Silva et al., 1999). 


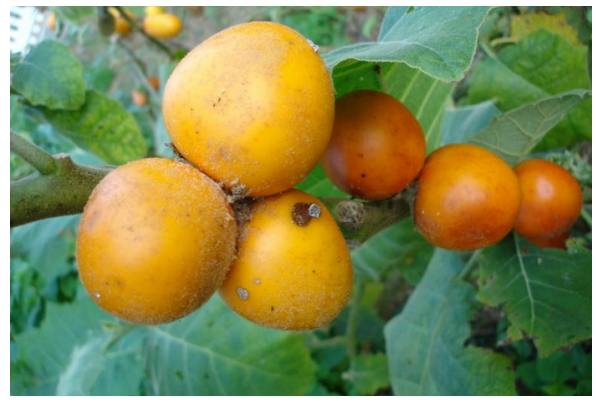

Figura 3: Cubiu (Solanum sessiliflorum D.). (Aires, 2012).

A Jurubeba (Solanum paniculatum L.) (Figura 4), por sua vez, está distribuída por toda a América tropical, é considerada uma planta invasora, perene, arbustiva, atingindo até $2 \mathrm{~m}$ de altura (Forni-Martins, Marques e Lemes, 1998). A esta espécie é frequentemente associada ao seu uso culinário além de diversos usos medicinais.

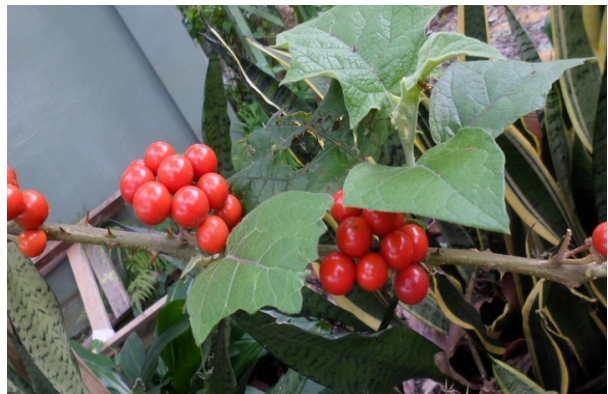

Figura 4: Jurubeba (Solanum paniculatum L.). (Aires, 2012).

Neste sentido, comparou-se os teores desses nutrientes encontrados nas PANC's consumidas nas comunidades ribeirinhas em estudo, com os valores encontrados nas plantas comumente consumidas da família Solanaceae e, com os valores recomendados de nutrientes diário da ANVISA (ANVISA, 2004). No sentido de avaliar a possibilidade de uma orientação nutricional baseadas em princípios de desenvolvimento local e diversificação da alimentação (NEPA/UNICAMP, 2011).

As três espécies Camapu (Physalis angulata L.), Cubiu (Solanum sessiliflorum D.) e a Jurubeba (Solanum paniculatum L.) da família da Solanaceae, coletadas na região do baixo rio Branco tiveram o teor de Ferro (Fe) e Cálcio (Ca) avaliados e os resultados são apresentados na tabela 01.

Tabela 1: Teores médios de cálcio e ferro encontrado nas espécies Camapu, Jurubeba e Cubiu da família Solanaceae, na matéria seca, coletadas na região do baixo rio Branco, Roraima.

\begin{tabular}{|c|c|c|c|c|}
\hline \multirow[b]{2}{*}{$\begin{array}{c}\text { Minera } \\
I\end{array}$} & \multicolumn{3}{|c|}{${ }^{1}$ (média)----g kg } & \multirow{2}{*}{$\begin{array}{l}\mathrm{g} \mathrm{d}^{-1} \\
\mathrm{IDR}^{*}\end{array}$} \\
\hline & $\begin{array}{c}\text { Canapu } \\
\text { Fruto }\end{array}$ & $\begin{array}{c}\text { Jurubeba } \\
\text { Fruto }\end{array}$ & $\begin{array}{l}\text { Cubiu } \\
\text { Folha }\end{array}$ & \\
\hline $\mathrm{Ca}$ & $62,4 \pm 2,9$ & $48,7 \pm 2,1$ & $15,6 \pm 1,1$ & $1^{* *}$ \\
\hline $\mathrm{Fe}$ & $\begin{array}{c}1,52 \pm 0,1 \\
2\end{array}$ & $\begin{array}{c}0,17 \pm 0,0 \\
4\end{array}$ & $\begin{array}{c}0,28 \pm 0,0 \\
6\end{array}$ & $\underset{*}{0,014^{*}}$ \\
\hline
\end{tabular}

${ }^{* *}$ ANVISA, 2004

Observando os valores apresentados na tabela acima, percebe-se que o Canapu apresentou os maiores teores para cálcio $(\mathrm{Ca})$ e ferro (Fe) quando comparado ao Jurubeba e ao Cubiu. Ao comparar-se com os valores da ingestão recomendada, verifica-se que todas as espécies avaliadas apresentaram teores bem acima do valor diário recomendado pela ANVISA (ANVISA, 2004).

Neste sentido, comparou-se os teores desses nutrientes encontrados nas PANC's consumidas nas comunidades ribeirinhas em estudo, com os valores encontrados nas plantas comumente consumidas da família Solanaceae e, com os valores recomendados de nutrientes diário da ANVISA (ANVISA, 2004). No sentido de avaliar a possibilidade de uma orientação nutricional baseadas em princípios de desenvolvimento local e diversificação da alimentação (NEPA/UNICAMP, 2011).

O teor de Ferro encontrado nas PANC's mostrou-se superior àquele encontrado em plantas convencionais, sendo que Physalis angulata apresentou um teor dez vezes superior a Solanum lycopersicum (Figura 5). Solanum sessiflorum e Solanum paniculatum apresentaram um teor equivalente a Solanum lycopersicum, porém muito superiores às demais plantas convencionais.

Nos teores de cálcio pode-se observar que o valor do Solanum lycopersicum é 1,2 vezes maior do que o Solanum sessiflorum (Figura 6), nota-se que somente este apresenta um valor superior à de uma PANC. Mas comparando o Solanum gilo com Solanum sessiflorum (fruto) obtém-se uma diferença de 627 vezes. O cálcio se apresenta em pequenas quantidades nas plantas Solanum gilo, Solanum 
melongena, Capsicum annuum e Solanum tuberosum, e com isso nota-se a abundância desse mineral nas PANC's, podendo preencher expressivamente a falta de nutrientes com relação ao acesso às plantas convencionais.

Em comparação com os valores recomendados para ingestão de nutrientes diários na dieta de um adulto a ANVISA sugere $14 \mathrm{mg} /$ dia de Ferro e 1 $\mathrm{g} /$ dia de Cálcio. Para o $S$. sessiflorum (fruto), (a perda dos frutos durante o armazenamento em campo fez com que a análise comparativa fosse feita por meio das informações disponíveis na literatura), a quantidade recomendada para ingestão é de $11,3 \mathrm{~g}$ da fruta por dia para atingir os valores referentes à ANVISA (2004) acima citados, sendo estes para cálcio + ferro (ANVISA, 2004). Notase pelas figuras 5 e 6 que 0 fruto do $S$. sessiflorum é abundante quando se trata desses dois minerais. O mesmo cálculo foi utilizado para as demais plantas aqui citadas, (sendo o valor total mencionado de cálcio e ferro em gramas da fruta de cada planta diariamente) para $\circ P$. angulata recomenda-se ingerir cerca de $25,3 \mathrm{~g}$, e para a S. paniculatumi, 102,88g.

Para as plantas consumidas comumente tem-se os seguintes valores: $S$. tuberosum 2,85 $\mathrm{Kg}$; S. gilo $5,1 \mathrm{Kg}$; S. melongena $16,09 \mathrm{Kg} ; \mathrm{S}$. lycopersicum $0,005 \mathrm{~kg}$ e C. annuum $13,5 \mathrm{Kg}$. Ressalta-se que uma alimentação balanceada não contém somente um tipo de alimento, então os valores devem ser diminuídos e a dieta diversificada.

Nota-se a abundância de $\mathrm{Ca}$ e $\mathrm{Fe}$ encontrado nas PANC's, os teores aqui avaliados, desses nutrientes são altos quando comparados com as plantas mais consumidas, porém se os frutos forem consumidos moderadamente, como nas quantidades descritas acima, não apresentariam risco de intoxicação desses minerais.

Os dois nutrientes citados são de suma importância para o bom funcionamento do organismo, porém em grandes quantidades podem se tornar tóxicos, assim como todos os demais nutrientes.

\section{CONCLUSÕES:}

Das três espécies de PANC's coletadas, com exceção do cubiu, que é esporadicamente cultivado, as demais foram coletadas em região de mata e sem cultivo. $E$ as análises realizadas mostraram que os teores de $\mathrm{Ca}$ de $\mathrm{Fe}$, nestas plantas, encontram-se dentro do recomendado pela ANVISA, sendo indicado o consumo dessas plantas no que se trata desses minerais. Ainda, percebe-se que, por possuírem uma maior concentração desses nutrientes, a quantidade consumida para atingir os valores diários recomendados é consideravelmente menor quando comparados com consumo necessário das plantas convencionais utilizadas nesse estudo

A incrementação de PANC's na alimentação cotidiana pode ser uma alternativa no que se refere a segurança nutricional disponível para as comunidades ribeirinhas, bem como o uso e propagação dessas plantas pode ser um meio de preservar a vegetação típica daquele local. Não obstante, a diversidade alimentar à mesa reflete em uma maior diversidade nas áreas de cultivo, garantindo a manutenção de agroecossistemas mais equilibrados, ainda mais quando se tratam de plantas nativas e espontâneas.

\section{AGRADECIMENTOS:}

Ao CNPq pelo financiamento da pesquisa (Processo $n^{\circ}$. 564343/2010-2). À EPAGRI-SC pelo apoio no processamento das amostras (Convênio $n^{\circ}$. 266/2012). À Universidade Estadual de Roraima, por meio do Laboratório de Turismo Ecologia e Meio Ambiente, pelo apoio logístico.

\section{REFERÊNCIAS:}

1. Albuquerque. U. P. Introdução à Etnobotânica - Recife: Bagaço, 2002. 87p

2. Amorozo, M. C. M.; Viertler, R. B. Abordagem qualitativa na coleta e análise de dados em etnobiologia e etnoecologia. In: Albuquerque, U. P.; Lucena, R. F. P.; Cunha, C. L. V. F. 2002. Métodos e Técnicas na Pesquisa Etnobiológica e Etnoecológica. Recife: NUPEEA, p: $65-$ 82.

3. Anvisa (Agência Nacional de Vigilância Sanitária). Regulamento Técnico sobre Ingestão Diária Recomendada (Idr) para 
Proteína, Vitaminas e Minerais. Consulta Pública $n^{\circ} 80$, de 13/12/2004.

4. Brasil. Manual de hortaliças nãoconvencionais, Ministério da Agricultura, Pecuária e Abastecimento; Brasília, 2010.

5. Ferreira, G. C.; Andrade, A. C. S. Diretrizes para coleta, herborização e identificação de material botânico nas Parcelas Permanentes em florestas naturais da Amazônia brasileira. ManausAM, 2006.

6. Forni-Martins, E. R.; Marques, M. C. M.; Lemes, M. R. Biologia floral e reprodução de Solanum paniculatum L. (Solanaceae) no estado de São Paulo, Brasil. Revista Brasileira de Botânica, São Paulo, v. 21, n. 2 ,

1998.

(http://www.scielo.br/scielo.php?

script=sci_arttext\&pid=S01008404199800 $0200002 \&$ Ing=en\&nrm=iso) Acesso em: 30/11/2014.

7. Lorenzi. H.; Matos. F. J. A. 2002. Plantas Medicinais no Brasil: nativas e exóticas cultivadas. Nova Odessa, São Paulo: Instituto Plantarum. 2002.

8. Nepa/Unicamp; Taco. 2011. Tabela Brasileira de Composição de Alimentos. 4ta ed. FODEPAL, Campinas - SP, 2011, $164 p$.

9. Padulosi, S.; Hoeschle-Zeledon, I. A qué denominamos especies subutilizadas.
Leisa Revista de Agroecologia, 2004, p: 6-8.

10. Rapport, E. H.; Marzocca, A. \& Drausal, B. S. Malezas comestibles del cono sur y otras partes del planeta. Fundación Normatil, 2009.

11. Raven, P. H., Evert, R. F., Eichhorn, E. S. Biologia Vegetal. 5ed. Rio de Janeiro: Guanabara Koogan, 1996, 738p.

12. Silva Filho, D.F.; Andrade, J.S.; Clement, C.R.; Machado, F.M.; Noda, H. Correlações fenotípicas, genéticas e ambientais entre descritores morfológicos e químicos em frutos de cubiu (Solanum sessiliflorum Dunal) da Amazonia. Acta Amazônica, 1999. 29: 503-511.

13. Stefanello, S. et al. Amadurecimento de frutos de cubiu (Solanum sessiliflorum Dunal) tratados com Etefon. Acta Amazônica. 2010. 40: 424-434.

14. Verdejo, M. E. 2006 - Diagnóstico Rural Participativo - Um guia prático- Brasília, 2006.

15. Yuyama, L. K. O.; Macedo, S. H. M.; Aguiar, J. P. L.; Filho, D. S.; Yuyama, K.; Fávaro, D. I. T; Vasconcellos, M. B. A. 2007. Quantificação de macro e micro nutrientes em algumas etnovariedades de cubiu (Solanum sessiliflorum Dunal). Acta Amazônica, 2007, 37: 425 - 430.

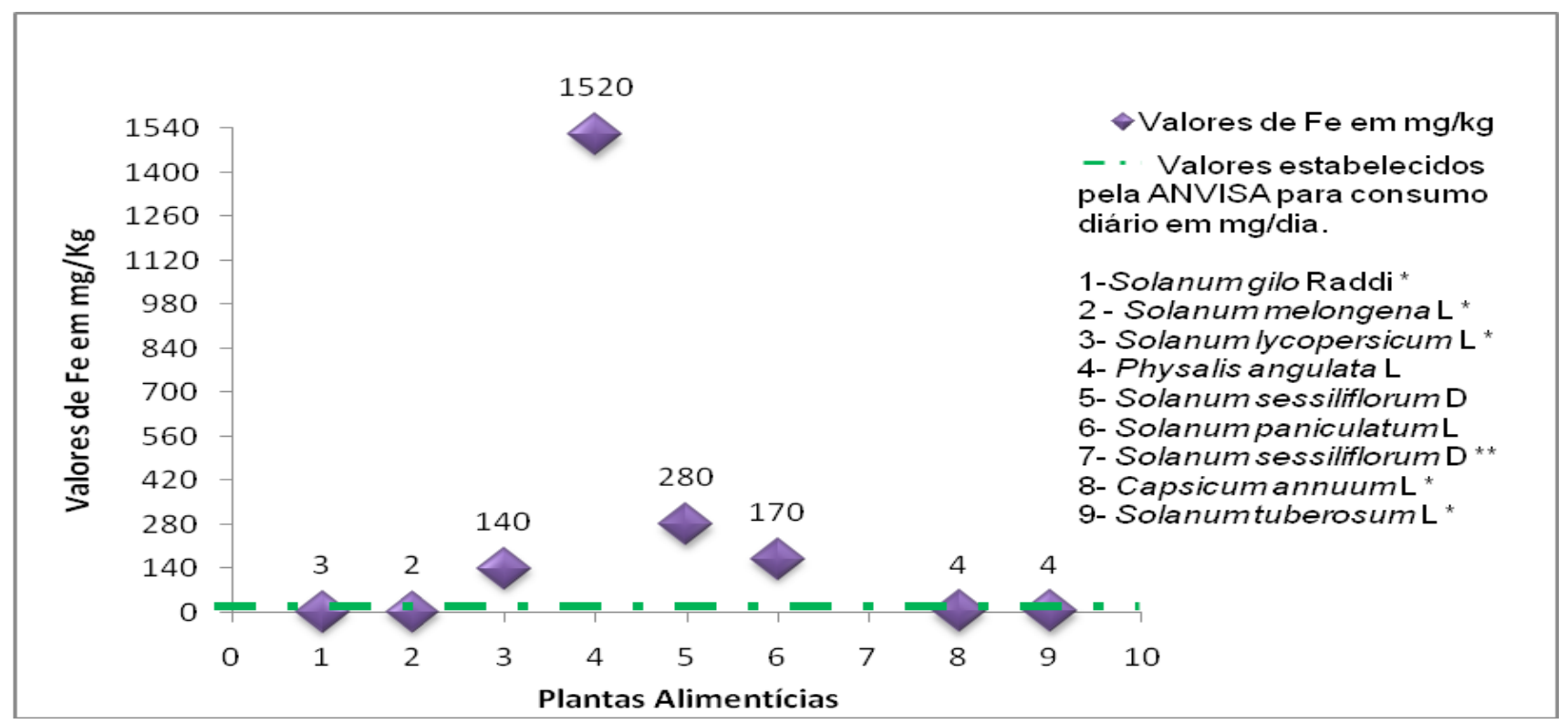

Figura 05: Teor de Fe nas PANC's e convencionais. * Valores encontrados na TACO - Tabela Brasileira de Composição de Alimentos (NEPA/UNICAMP, 2011); ** Valores disponibilizados (Yuyama et al., 2007).

PERIÓDICO TCHÊ QUÍMICA • www.periodico.tchequimica.com • Vol. 13 N. 25.

- ISSN 1806-0374 (impresso) • ISSN 1806-9827 (CD-ROM) • ISSN 2179-0302 (meio eletrônico) 


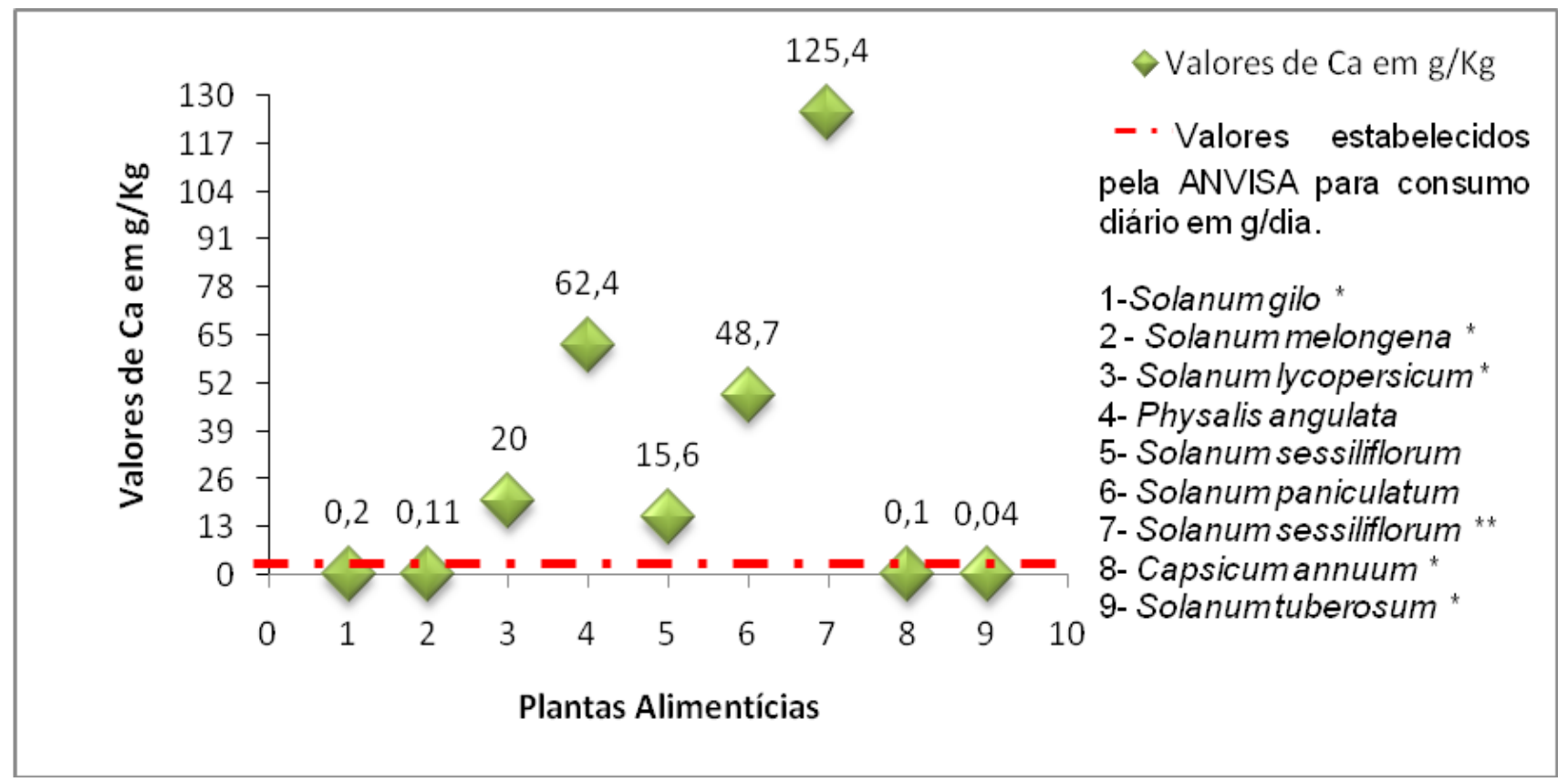

Figura 6: Teor de Ca encontrado nas PANC's e convencionais. * Valores encontrados na TACO - Tabela Brasileira de Composição de Alimentos (NEPA/UNICAMP, 2011). ** Valores disponibilizados (Yuyama et al., 2007).

PERIÓDICO TCHÊ QUÍMICA • www.periodico.tchequimica.com • Vol. 13 N. 25.

• ISSN 1806-0374 (impresso) • ISSN 1806-9827 (CD-ROM) • ISSN 2179-0302 (meio eletrônico)

$$
\text { (C) 2015. Porto Alegre, RS. Brasil }
$$

The Periódico Tchê Química (ISSN: 1806-0374; 2179-0302) is an open-access journal since 2004. Journal DOI: 10.52571/PTQ. http://www.tchequimica.com. This text was introduced in this file in 2021 for compliance reasons.

() The Author(s)

OPEN ACCESS. This article is licensed under a Creative Commons Attribution 4.0 (CC BY 4.0) International License, which permits use, sharing, adaptation, distribution, and reproduction in any medium or format, as long as you give appropriate credit to the original author(s) and the source, provide a link to the Creative Commons license, and indicate if changes were made. The images or other third-party material in this article are included in the or exceeds the permitted use, you will need to obtain permission directly from the copyright holder. To view a copy of this license, visit http///creativecommons. org/licenses/by/4.0/. 